\title{
On Asymptotic Behavior of Zeta Singularities for Compact Locally Symmetric Spaces
}

\author{
DŽENAN GUŠIĆ \\ University of Sarajevo \\ Faculty of Sciences and Mathematics \\ Department of Mathematics \\ Zmaja od Bosne 33-35, 71000 Sarajevo \\ BOSNIA AND HERZEGOVINA \\ dzenang@pmf.unsa.ba
}

\begin{abstract}
We obtain precise estimates for the number of singularities of Selberg's and Ruelle's zeta functions for compact, higher-dimensional, locally symmetric Riemannian manifolds of strictly negative sectional curvature. The methods applied in this research represent a generalization of the methods described in the case of a compact Riemann surface. In particular, this includes an application of the Phragmen-Lindelof theorem, the variation of the argument of certainzetafunctions, aswellastheuseofsomeclassicalanalyticnumbertheorytechniques.
\end{abstract}

Key-Words: Phragmen-Lindelof theorem, zeta functions, distrubution of singularities, compact locally symmetric spaces,countingfunctions

Received: March 27, 2020. Revised: September 17, 2020. Accepted: September 27, 2020. Published: October 13, 2020.

\section{Introduction}

Suppose that $\mathcal{K}$ is a compact Riemann surface.

As it is known, $\mathcal{K}$ may be represented as a quotient of the upper half plane $H^{+}$by a discontinuous group $\Gamma$.

It is assumed that $H^{+}$is endowed with the metric $y^{-2}\left((d x)^{2}+(d y)^{2}\right)$.

Denote by $A$ the volume of $\mathcal{K}$.

As it is also known, the Riemann hypothesis for the Selberg zeta function in this setting is almost true, which means that any possible exceptional zeros are all located in the real interval $(0,1)$.

In this scenario, the Selberg zeta function $Z(s)$ is for $\operatorname{Re}(s)>1$, defined by the product

$$
\prod_{\gamma} \prod_{n=0}^{\infty}\left(1-e^{-l_{\gamma}(s+n)}\right)
$$

where $l_{\gamma}$ runs through the set of lengths of primitive closed geodesics on $\mathcal{K}$.

The known fact is that $Z(s)$ is uniformly bounded and bounded away from zero in any half-plane of the form $\operatorname{Re}(s)>1+\varepsilon(\varepsilon>0)$.

Applying the fact that $Z(s)$ is of order 2, the Phragmén-Lindelöf theorem, and the functional equation for $Z(s)$, the author in [1], proved that

$$
Z(\sigma+\mathrm{i} t)=e^{O(|t|)},
$$

uniformly in any bounded strip $b_{1} \leq \sigma \leq b_{2}$.

There, the number of zeros of $(Z(s))^{2}$ in the rectangle $R$, defined by the inequalities $1-a \leq \operatorname{Re}(s) \leq$ $a,-T \leq \operatorname{Im}(s) \leq-T$, where $a$ is a positive number satisfying $1<a<{ }_{4}^{5}$, and $T$ is selected so that no zero occurs on the boundary of $R$, is denoted by $2 N(T)$.

Consequently, the number of zeros of $Z(s)$ itself in this rectangle is $N(T)$.

Thus, it is proved that

$$
N(T)=\frac{\overline{A T^{2}}}{2 \pi}+4 S(T)+O(1)
$$

where

$$
S(T)={ }_{\pi}^{\overline{1}} \operatorname{Im}\left(\int_{C} Z^{\prime}(s) d s\right),
$$

the $O(1)$ term is continuous and tends to a limit as $T$ $\rightarrow \infty, S(T)$ is the ${ }_{\pi}^{1}$ times the variation of the argument of $Z(s)$ along $C$, where $C$ is the portion of $\partial R$ consisting of the vertical segment from- $a$ to i $T$, plus the horizontal segment from $a+\mathrm{i} T$ to ${ }_{2}^{1}+\mathrm{i} T(S(T)$ plays the same role in this setting as does its counterpart from the theory of the Riemann zata function).

By [1, p. 212, Lemma 3], $S(T)=O(T)$.

Moreover, by Theorem 1 in [1, p. 216] (see also, [2], [3]), $S(T)=O\left(T(\log T)^{-1}\right)$. 
The main purpose of this research is to derive analogous results of the results described above, for the case of compact, higher-dimensional, locally symmetric Riemannian manifolds of strictly negative sectional curvature. In other words, our goal is to derive asymptotic formulas for the number of singularities of the zeta functions naturally attached to this setting. The singularities of these zeta functions are described by Theorem 3.15 in [4, p. 113]. Thus, the singularities are generated by the eigenvalues of the corresponding differential operators. This means that the knowledge about the number of the singularities implies the knowledge about the number of the corresponding eigenvalues (the Weyl law) and vice versa. Note that the locally symmetric spaces in this research are compact ones. Assume for a moment that $S$ is a Riemannian symmetric space of noncompact type, with the gruop of motions $G$ of $S$. Then the algebra $L_{D i f f}(S)$ of $G$-invariant differential operators on $S$ is comutative, and its spectrum $\Lambda(S)$ can be canonically identified with $F / w$, where $F$ is a complex vector space with dimension equal to the rank of $S$, and $w$ is a finite subgroup of $G L(F)$ generated by reflexions. Let $\Gamma$ be a discrete subgroup of $G$ that acts freely on $S$, and let $X=\Gamma \backslash S$. Then (see [5]), the members of $L_{D i f f}(S)$ may be regarded as differential operators on $X$. Let us now assume that $X$ is compact, and define the spectrum $\Lambda$ of $X$ as the set of those elements of $\Lambda(S)$ for which one can find a nonzero eigenfuncion defined on $X$. By Theorem 9.1 in [5, p. 89], the Weyl asymptotic law states that

$$
\begin{aligned}
& \sum_{\lambda \in \Lambda_{p},\|\lambda\| \leq t} m(\lambda) \\
= & (2 \sqrt{\pi})^{-n} \Gamma\left(\frac{n}{2}+1\right) \operatorname{vol}(X) t^{n}+ \\
& O\left(t^{n-1}(\log t)^{-1}\right)
\end{aligned}
$$

as $t \rightarrow+\infty$, where $m(\lambda)$ is the multiplicity of $\lambda \in \Lambda$, and $\Lambda_{p}$ is the principal spectrum of $\Lambda$. Having in mind this result and Theorem 3.15 in [4], we know that the number of desired singularities in this research must be of the form (1). However, our goal is to derive the formulas explicitly (with the constants determined explicitly) by following the classical approach of Randol [1] in the case of compact Riemann surfaces. Thus, the results derived in this paper will confirm the expected form (1) with the leading coefficients determined explicitly (which is the fact that might be used very well in the future research, like during deriving of approximate formulas for the logarithmic derivative of the zeta functions, during estimation of the error terms in the prime geodesic therems, etc).

\section{Preliminaries}

Through the rest of the paper, we assume that $Y$ is a compact, $n$-dimensional, locally symmetric Riemannian manifold of strictly negative sectional curvature.

As it is known, $Y$ can be represented in the form $Y=\Gamma \backslash G / K$, where $G$ is a connected semi-simple Lie group of real rank one, $K$ is the maximal compact subgroup of $G$, and $\Gamma$ is a discrete, co-compact, and torsion-free subgroup of $G$.

Let $\mathfrak{a} \subset \mathfrak{p}$ be a one-dimensional subspace of $\mathfrak{p}$, where $\mathfrak{p}$ is defined by the Cartan decomposition $\mathfrak{g}=\mathfrak{k}$ $\oplus \mathfrak{p}$ of the Lie algebra $\mathfrak{g}$ of $G$.

If $G_{a}$ is the centralizer of $a$ in $G$, then we define $M$ by $M=G_{a} \cap K$.

We have the Iwasawa decomposition $G=K A N$ and the corresponding decomposition $\mathfrak{g}=\mathfrak{k} \oplus \mathfrak{a} \oplus \mathfrak{n}$, where $\mathfrak{n}$ is the sum of the corresponding positive root spaces $[4$, p. 18].

As it is usual, we put $A^{+}$to be $\exp \left(\mathfrak{a}^{+}\right) \subset A$, and $W=W(\mathfrak{g}, \mathfrak{a})$ to be the Weyl group.

Let $X_{d}$ be the compact dual space of $X=G / K$, the Riemannian symmetric space of rank one.

The following classical result will be applied in the sequel [6, p. 138].

Theorem 1. (Phragmén-Lindelöf Theorem) Let G be a simply connected region and let $f$ be an analytic function on $G$. Suppose that there is an analytic function $\varphi: G \rightarrow \mathbb{C}$ which never vanishes and is bounded on $G$. If $M$ is a constant and $\partial_{\infty} G=A \cup B$ such that:

(a) for every $a \in A, \limsup _{z \rightarrow a}|f(z)| \leq M$,

(b) for every $b \in B$, and $\eta>0$, $\limsup _{z \rightarrow b}|f(z)||\varphi(z)|^{\eta}$ $\leq M$,

then, $|f(z)| \leq M$ for all $z \in G$.

\section{Results}

Theorem 2. Functions $Z_{S, \chi}(s, \sigma), S_{\chi}(s, \sigma)$ and $S_{\chi}^{S}(s, \sigma)$ are bounded and bounded away from zero for $\operatorname{Re}(s)>\rho$. Furthermore, functions $Z_{R, \chi}(s, \sigma)$ and $Z_{R, \chi}^{S}(s, \sigma)$ are bounded and bounded away from zero for $\operatorname{Re}(s)>2 \rho$, where $Z_{S, \chi}(s, \sigma)$ and $S_{\chi}(s, \sigma)$ are defined on page 97, $S_{\chi}^{S}(s, \sigma)$ is defined on page 98, $Z_{R, \chi}(s, \sigma)$ is defined on page 96 , and $Z_{R, \chi}^{S}(s, \sigma)$ is defined on page 133 in [4], respectively. Furthermore, $\rho, \chi$ and $\sigma$ are introduced on pages 18 and 95 of the same book. 
Proof. The notation $S$ resp. $R$ is used to denote the Selberg resp. the Ruelle zeta function.

$s$ is a complex variable, and $\mathbf{S}$ denotes the super zeta function.

Suppose that $n$ is an even integer.

Taking into account Proposition 3.4 in [4, p. 99], we see that it will suffice to prove the result for $Z_{S, \chi}(s, \sigma)$.

We have that,

$$
\log Z_{S, \chi}(s, \sigma)
$$$$
=(-1)^{n} \sum_{\gamma \in \Gamma_{h}} \frac{\operatorname{tr} \sigma(m) \operatorname{tr} \chi(\gamma)}{\operatorname{det}\left(1-\operatorname{Ad}(m a)_{\mathfrak{n}}\right) n_{\Gamma}(\gamma)} e^{(\rho-s) l(\gamma)},
$$

where $\Gamma_{h}$ is the set of all $\Gamma$-conjugacy classes of hyperbolic elements in $\Gamma, n_{\Gamma}(\gamma)$ (for $g \in \Gamma$ ) is the number of classes in $\Gamma_{g} /\langle g\rangle$, where $\Gamma_{g}$ is the centralizer of $g$ in $\Gamma$, and $\langle g\rangle$ is the group generated by $g, l(g)$ (for $g \in \Gamma$ ) is the length of the closed geodesic of $Y$ corresponding to $g$, and $\gamma \in \Gamma_{h}$ has the form $\gamma=a m$ $=a_{\gamma} m_{\gamma} \in A^{+} M$ (see, e.g., [7], [8]).

Put,

$$
C_{\chi}(g, \sigma)=\operatorname{tr} \chi(g) C(\gamma, \sigma),
$$

where

$$
C(\gamma, \sigma)=(-1)^{n-1} \frac{l(g) \operatorname{tr} \sigma(m)}{2 \operatorname{det}\left(1-\operatorname{Ad}(m a)_{\mathfrak{n}}\right)} e^{\rho l(g)} .
$$

It follows that,

$\log Z_{S, \chi}(s, \sigma)=-2 \times$

$$
\begin{aligned}
& \sum_{\gamma \in \Gamma_{h}} \frac{(-1)^{n-1} \operatorname{tr} \sigma(m) \operatorname{tr} \chi(\gamma) l(\gamma)}{2 \operatorname{det}\left(1-\operatorname{Ad}(m a)_{\mathfrak{n}}\right) n_{\Gamma}(\gamma) l(\gamma)} e^{(\rho-s) l(\gamma)} \\
& =-2 \sum_{\gamma \in \Gamma_{h}} \frac{C_{\chi}(\gamma, \sigma)}{n_{\Gamma}(\gamma) l(\gamma)} e^{-s l(\gamma)}
\end{aligned}
$$

Put,

$$
\pi_{\Gamma}^{1}(x)=\#\left\{\gamma \in \Gamma_{h}: l(\gamma) \leq x\right\} .
$$

It is known that there exist constants $C^{\prime}$ and $C^{\prime \prime}$, such that

$$
C_{\chi}(g, \sigma) \leq C^{\prime} e^{-\rho l(g)}
$$

and

$$
\pi_{\Gamma}^{1}(x) \leq C^{\prime \prime} e^{2 \rho x}
$$

Suppose that $\sigma_{1}>\rho$, where $\sigma_{1}=\operatorname{Re}(s)$.

Now,

$$
\begin{aligned}
& \left|\log Z_{S, \chi}(s, \sigma)\right| \\
\leq & 2 C^{\prime} \sum_{\gamma \in \Gamma_{h}} \frac{e^{-\rho l(\gamma)}}{l(\gamma)} e^{-\sigma_{1} l(\gamma)} \\
= & 2 C^{\prime} \sum_{\substack{\gamma \in \Gamma_{h} \\
0<l(\gamma)<1}} \frac{e^{-\rho l(\gamma)}}{l(\gamma)} e^{-\sigma_{1} l(\gamma)}+ \\
& 2 C^{\prime} \sum_{\gamma \in \Gamma_{h}} \frac{e^{-\rho l(\gamma)}}{l(\gamma)} e^{-\sigma_{1} l(\gamma)} \\
= & O(1)+O\left(\sum_{\gamma \in \Gamma_{h}} e^{-\rho l(\gamma)} e^{-\sigma_{1} l(\gamma)}\right. \\
= & O\left(\int_{1}^{+\infty} e^{-\rho t} e^{-\sigma_{1} t} d \pi_{\Gamma}^{1}(t)\right) \\
= & O\left(\int_{1}^{+\infty} e^{-\rho t} e^{-\sigma_{1} t} e^{2 \rho t} d t\right) \\
= & O\left(\int_{1}^{+\infty} e^{\left(\rho-\sigma_{1}\right) t} d t\right)=O(1) .
\end{aligned}
$$

On the other hand,

$\log Z_{S, \chi}(s, \sigma)^{-1}=2 \sum_{\gamma \in \Gamma_{h}} \frac{C_{\chi}(\gamma, \sigma)}{n_{\Gamma}(\gamma) l(\gamma)} e^{-s l(\gamma)}$.

Hence,

$$
\left|\log Z_{S, \chi}(s, \sigma)^{-1}\right| \leq 2 C^{\prime} \sum_{\gamma \in \Gamma_{h}} \frac{e^{-\rho l(\gamma)}}{l(\gamma)} e^{-\sigma_{1} l(\gamma)}
$$

for $\sigma_{1}>\rho$.

This completes the proof in this case (see also, [9,

Suppose that $n$ is an odd integer.

Taking into account the equations:

$$
\begin{aligned}
S_{\chi}(s, \sigma) & =Z_{S, \chi}(s, \sigma) Z_{S, \chi}(s, w \sigma), \\
S_{\chi}^{\mathbf{S}}(s, \sigma) & =\frac{Z_{S, \chi}(s, \sigma)}{Z_{S, \chi}(s, w \sigma)}, \\
Z_{R, \chi}^{\mathbf{S}}(s, \sigma) & =\frac{Z_{R, \chi}(s, \sigma)}{Z_{R, \chi}(s, w \sigma)},
\end{aligned}
$$


where $w \in W$ is a non-trivial element, we see that it will suffice to prove the result for $Z_{S, \chi}(s, \sigma)$.

However, the result for $Z_{S, \chi}(s, \sigma)$ follows in exactly the same way as in the even-dimensional case.

This completes the proof.

Theorem 3. For zeta functions $Z_{s, \chi}(s, \sigma)$ and $S_{\chi}(s, \sigma)$, we have

$$
\left|Z_{i}\left(\sigma_{i}+\mathrm{i} t\right)\right|=e^{O\left(|t|^{n-1}\right)},
$$

$i=1,2$, uniformly in any bounded strip $b_{1} \leq \sigma_{1} \leq b_{2}$, where $Z_{1}(s)$ and $Z_{2}(s)$ are entire functions of order at most $n$, such that

$$
f(s)=\frac{Z_{1}(s)}{Z_{2}(s)}
$$

for $f(s) \in\left\{Z_{S, \chi}(s, \sigma), S_{\chi}(s, \sigma)\right\}$ (see, [10, p. 528, Th. 4.1. (b)] in the even-dimansional case, and [11, p. 306, Th. 2.] in the odd-dimensional case).

Proof. Suppose that $n$ is an even number.

Let $c>\max \left\{\rho,\left|b_{1}\right|,\left|b_{2}\right|\right\}$.

It is enough to prove the assertion for a wider strip $-c \leq \sigma_{1} \leq c$.

So far, we have that $Z_{1}(s)$ and $Z_{2}(s)$ are of finite order at most $n$.

$Z_{S, \chi}(s, \sigma)$ converges for $\operatorname{Re}(s)>\rho$.

By Theorem 2, $Z_{S, \chi}(s, \sigma)$ is bounded and bounded away from zero on $\operatorname{Re}(s)=c$.

On $\operatorname{Re}(s)=-c$, we apply the functional equation from Theorem 10 in [11, p. 318].

Notice that $g(t)$ in the functional equation is a polynomial of degree $n-1$.

Thus,

$$
\left|Z_{S, \chi}(-c+\mathrm{i} t, \sigma)\right|=e^{O\left(|t|^{n-1}\right)} .
$$

Now, the assertion of theorem (in this case), follows by application of Phragmén-Lindelöf theorem [6, Theorem VI. 4.1.] (Cf. also [9, p. 60] in the quartic field setting).

Note that we take $c>\rho$, since for $\sigma_{1}>\rho$, the Selberg zeta function $Z_{S, \chi}(s, \sigma), s=\sigma_{1}+\mathrm{i} t$ is bounded.

By previously applied functional equation, we have that

$$
\left|Z_{S, \chi}(s, \sigma)\right|=|f(t)| e^{g(t)}\left|Z_{S, \chi}(-s, \sigma)\right| .
$$

Therefore,

$$
\begin{aligned}
& \log \left|Z_{S, \chi}(s, \sigma)\right| \\
= & \log |f(t)|+g(t)+\log \left|Z_{S, \chi}(-s, \sigma)\right| .
\end{aligned}
$$

Since $f(t)$ is bounded function, it follows that $\log |f(t)|$ is also bounded.

If $\sigma_{1}=-c$, then $\operatorname{Re}(-s)>\rho$, so $Z_{S, \chi}(-s, \sigma)$ is bounded for $\sigma_{1}=-c$.

Consequently, $\log \left|Z_{S, \chi}(-s, \sigma)\right|$ is bounded for $\sigma_{1}=-c$.

Now, (2), and the degree of $g(t)$, yield that $\log \left|Z_{S, \chi}(s, \sigma)\right|=O\left(|t|^{n-1}\right)$ for $\sigma_{1}=-c$.

It follows that $Z_{S, \chi}(s, \sigma)=e^{O\left(|t|^{n-1}\right)}$ for $\sigma_{1}=$ $-c$.

Since $Z_{S, \chi}(s, \sigma)=\frac{Z_{1}(s)}{Z_{2}(s)}$, and $Z_{1}(s), Z_{2}(s)$ are entire functions of order at most $n$, we obtain that $Z_{i}(s)=e^{O\left(|t|^{n-1}\right)}$ for $\sigma_{1}=-c, i=1,2$.

Now, we prove that $\left|Z_{1}(s)\right|=e^{O\left(|t|^{n-1}\right)}$ for $-c$ $\leq \sigma_{1} \leq c$ (we apply the same reasoning for $Z_{2}(s)$ ).

We apply Phragmén-Lindelöf theorem (Theorem 1 ), where $G$ is given by $-c \leq \sigma_{1} \leq c, A$ is the line $-c$ $+\mathrm{i} t$, and $B$ is the line $c+\mathrm{i} t$.

Since $Z_{1}(s)=e^{O\left(|t|^{n-1}\right)}$ for $\sigma_{1}=-c$, we conclude that there exists a constant $C_{1}$, such that

$$
\frac{\left|Z_{1}(s)\right|}{e^{C_{1}|t|^{n-1}}} \leq 1=M_{1}
$$

for $\sigma_{1}=-c$.

Since the order of $Z_{1}(s)$ is at most $n$, we have that $Z_{1}(s)=e^{O\left(|t|^{n}\right)}$ for $\sigma_{1}=c$.

Thus, there is a constant $C_{2}$, such that

$$
\frac{\left|Z_{1}(s)\right|}{e^{C_{2}|t|^{n}}} \leq 1
$$

for $\sigma_{1}=c$.

Note that the function $\frac{1}{e^{C_{1}|t|^{n-1}}}$ is bounded on $G$.

In particular, it is bounded for $\sigma_{1}=c$.

Therefore, there exists a constant $M_{2}$, such that

$$
\frac{1}{e^{C_{1}|t|^{n-1}}} \leq M_{2}
$$

for $s \in G$.

In particular, (5) is satisfied for $\sigma_{1}=c$.

Now, we put $f(s)=\frac{Z_{1}(s)}{e^{C_{1}|t|^{n-1}}}, \varphi(s)=\frac{1}{e^{C_{2}|t|^{n}}}$ into Theorem 1.

Note that $f(s)$ is analytic function on $G$, while the function $\varphi(s)$ is analytic on $G$ (without zeros), and is bounded on $G$. 
The fact that $\varphi(s)$ is bounded on $G$ implies that there is a constant $K$, such that

$$
\varphi(s)=|\varphi(s)| \leq K
$$

for $s \in G$.

Now, if $\sigma_{1}=-c$, the inequality (3) yields that

$$
|f(s)| \leq M_{1}
$$

for $\sigma_{1}=-c$.

Thus, the condition $(a)$ in Theorem 1 is satisfied. If $\sigma_{1}=c$, then (4), (6) and (5), give us

$$
\begin{aligned}
& |f(s)||\varphi(s)|^{\eta} \\
= & \frac{\left|Z_{1}(s)\right|}{e^{C_{1}|t|^{n-1}}} \frac{1}{e^{\eta C_{2}|t|^{n}}} \\
= & \frac{\left|Z_{1}(s)\right|}{e^{C_{2}|t|^{n}}} \frac{1}{e^{-C_{2}|t|^{n}}} \frac{1}{e^{C_{1}|t|^{n-1}}} \frac{1}{e^{\eta C_{2}|t|^{n}}} \\
= & \frac{\left|Z_{1}(s)\right|}{e^{C_{2}|t|^{n}}} \frac{1}{e^{(\eta-1) C_{2}|t|^{n}}} \frac{1}{e^{C_{1}|t|^{n-1}}} \\
= & \frac{\left|Z_{1}(s)\right|}{e^{C_{2}|t|^{n}}}\left(\frac{1}{e^{C_{2}|t|^{n}}}\right)^{\eta-1} \frac{1}{e^{C_{1}|t|^{n-1}}} \\
= & \frac{\left|Z_{1}(s)\right|}{e^{C_{2}|t|^{n}}}(\varphi(s))^{\eta-1} \frac{1}{e^{C_{1}|t|^{n-1}}} \\
\leq & K^{\eta-1} M_{2} .
\end{aligned}
$$

Now, we proceed as in [6, p. 139].

The inequality (8) means that the condition $(b)$ in Theorem 1 is satisfied.

The inequalities (7) and (8) mean that the conditions $(a)$ and $(b)$ in Theorem 1, hold true (at the same time) for $M=\max \left\{M_{1}, K^{\eta-1} M_{2}\right\}$.

Thus, by Theorem 1, we have that $|f(s)| \leq$ $\max \left\{M_{1}, K^{\eta-1} M_{2}\right\}$ for $s \in G$.

If we let $\eta \rightarrow 1$ in the last inequality, we end up with $|f(s)| \leq \max \left\{M_{1}, M_{2}\right\}=M^{\prime}$ for $s \in G$.

In other words, $\frac{\left|Z_{1}(s)\right|}{e^{C_{1}|t|^{n-1}}} \leq M^{\prime}$ for $-c \leq \sigma_{1} \leq c$, i.e., $\left|Z_{1}(s)\right|=e^{O\left(|t|^{n-1}\right)}$ for $-c \leq \sigma_{1} \leq c$.

This completes the proof in this case.

Now suppose that $n$ is an odd integer.

Proof is the same as in the even-dimensional case.

In particular, we apply the functional equations from Theorems 11 and 12 in [11].

Note that $h(t)$ in these equations is a polynomial of degree $n-1$.

Thus,

$$
\begin{aligned}
\left|Z_{S, \chi}(-c+\mathrm{i} t, \sigma)\right| & =e^{O\left(|t|^{n-1}\right)}, \\
\left|S_{\chi}(-c+\mathrm{i} t, \sigma)\right| & =e^{O\left(|t|^{n-1}\right)} .
\end{aligned}
$$

Proceeding in exactly the same way as in the even-dimensional case, we obtain the claim.

This completes the proof.

Theorem 4. Let $n$ be an even integer. If $\gamma$ is $\sigma$ admissible, then

$$
\begin{aligned}
N(t)= & \frac{\operatorname{dim}(\chi) \chi(Y)}{\chi\left(X_{d}\right) T} \times \\
& \times \sum_{k=0}^{\frac{n}{2}-1}(-1)^{\frac{n}{2}-k} p_{n-2 k-1} \frac{t^{n-2 k}}{n-2 k}+ \\
& \frac{1}{\pi} S(t)+O(1)
\end{aligned}
$$

where the concept of admissible elements is introduced by [11, p. 305, Def. 1.], T and the coefficients $p_{n-2 k-1}$ are described by [11, p. 307, Lemma 4.], $N(t)$ denotes the number of singularities of $Z_{S, \chi}(s, \sigma)$ on the interval i $x, 0<x<t$, and $S(t)$ is the variation of the argument of $Z_{S, \chi}(s, \sigma)$ along C.

Here, $C$ denotes the portion of $\partial R$, consisting of the vertical segment from a to $a+\mathrm{i} t$ plus the horizontal segment from $a+\mathrm{i} t$ to $\mathrm{i} t$, where $R$ is the rectangle defined by the inequalities $-a \leq \operatorname{Re}(s) \leq a,-t$ $\leq \operatorname{Im}(s) \leq t$ for some $a>\rho$.

Let $n$ be an odd integer. Suppose that $r=1$ in the case (a) and $r=2$ in the case (b). Then,

$$
\begin{aligned}
N(t)= & \frac{1}{\pi} \sum_{k=0}^{\frac{n-1}{2}}(-1)^{\frac{n+1}{2}-k} c_{\frac{2 k-n}{2}} \Gamma\left(\frac{2 k-n}{2}\right) t^{n-2 k} \\
& +\frac{1}{\pi} S(t)+O(1)
\end{aligned}
$$

where the cases ( $a)$ and (b), and the coefficients $c_{\frac{2 k-n}{2}}$ are described by [11, p. 307, Lemma 5.], and $N^{2}(t)$ denotes the number of singularities $Z_{S, \chi}(s, \sigma)$ (case $(a)$ ) resp. $S_{\chi}(s, \sigma)$ (case (b)) on the interval i $x, 0<$ $x<t$, and $S(t)$ is the variation of the argument of $Z_{S, \chi}(s, \sigma)$ (case (a)) resp. $S_{\chi}(s, \sigma)$ (case (b)).

Here, $C$ is defined in the same way as in the evendimensional case.

Proof. Let $n$ be an even integer.

We adjust the argumentation from [1] to our situation, inserting some elements from [9] (see also, [12]).

Define,

$$
\xi(s)=\left(Z_{S, \chi}(s, \sigma)\right)^{2} e^{\phi(s)},
$$


where

$$
\begin{aligned}
& \phi(s) \\
= & K \int_{0}^{s} P_{\sigma}(w)\left\{\begin{array}{c}
\tan \left(\frac{\pi w}{T}\right), \\
-\cot \left(\frac{\pi w}{T}\right),
\end{array} \epsilon_{\sigma}=\frac{1}{2}\right. \\
K= & \frac{2 \pi \operatorname{dim}(\chi) \chi(Y)}{\chi\left(X_{d}\right) T} .
\end{aligned}
$$

$$
N(t)=\frac{1}{2 \pi} \operatorname{Im}\left(\int_{C} \frac{\xi^{\prime}(s)}{\xi(s)} d s\right)+O(1) .
$$

By (41) and (42) in [11, p. 318] (see also, [13]),

The polynomial $P_{\sigma}(w)$, and values $\epsilon_{\sigma} \in\left\{0, \frac{1}{2}\right\}$ are also introduced by Definition 1 in [11, p. 305].

Now, we specify $\phi(s)$ in the open upper and lower half-planes to be the value obtained by carrying out the integration along the line segment joining the origin to $s$.

Furthermore, if $\epsilon_{\sigma}=\frac{1}{2}$ resp. $\epsilon_{\sigma}=0$ and $s$ is on the real line, $s \neq \pm \frac{T}{2}, \pm \frac{3 T}{2}, \pm \frac{5 T}{2}, \ldots$ resp. $s \neq 0, \pm T$, $\pm 2 T, \ldots$, we define $\phi(s)$ by the requirement of continuity as $s$ is approached from the upper half-plane.

By $[11$, p. 308, eq. (6)],

$$
Z_{S, \chi}(-s, \sigma)=e^{\phi(s)} Z_{S, \chi}(s, \sigma)
$$

Hence,

$$
\begin{aligned}
\xi(-s) & =\left(Z_{S, \chi}(-s, \sigma)\right)^{2} e^{\phi(-s)} \\
& =\left(Z_{S, \chi}(s, \sigma)\right)^{2} e^{2 \phi(s)} e^{-\phi(s)} \\
& =\left(Z_{S, \chi}(s, \sigma)\right)^{2} e^{\phi(s)} \\
& =\xi(s) .
\end{aligned}
$$

As usual, $\xi(s)$ is real on the real axis and so $\overline{\xi(s)}$ $=\xi(\bar{s})$.

Assume that $t$ is selected so that no singularity of $Z_{S, \chi}(s, \sigma)$ occurs on the boundary of $R$.

We have,

$$
\begin{aligned}
& N(t) \\
= & \frac{1}{4} \cdot \frac{1}{2 \pi \mathrm{i}} \int_{\partial R} \frac{\xi^{\prime}(s)}{\xi(s)} d s-\frac{1}{2} N_{0} \\
= & \frac{1}{4} \cdot \frac{1}{2 \pi} \operatorname{Im}\left(\int_{\partial R} \frac{\xi^{\prime}(s)}{\xi(s)} d s\right)-\frac{1}{2} N_{0},
\end{aligned}
$$

where $N_{0}=O(1)$ is the number of singularities of $Z_{S, \chi}(s, \sigma)$ on the real line.

From the functional equation for $\xi(s)$ and the fact that $\overline{\xi(s)}=\xi(\bar{s})$, one obtains in a classical way that

$$
\begin{aligned}
& \phi\left(\sigma_{1}+i t\right) \\
= & \sum_{k=0}^{\frac{n}{2}-1} p_{n-2 k-1} \frac{t}{|t|} \frac{K \mathrm{i}}{n-2 k} \times \\
& \times \sum_{l=0}^{\frac{n}{2}-k}\left(\begin{array}{c}
n-2 k \\
2 l
\end{array}\right)(-1)^{l} \sigma_{1}^{n-2 k-2 l} t^{2 l}+ \\
& \sum_{k=0}^{\frac{n}{2}-1} p_{n-2 k-1} \frac{K}{n-2 k} \times \\
& \times \sum_{l=1}^{\frac{n}{2}-k}\left(\begin{array}{c}
n-2 k \\
2 l-1
\end{array}\right)(-1)^{l} \sigma_{1}^{n-2 k-2 l+1}|t|^{2 l-1} \\
& +O(1) .
\end{aligned}
$$

Now,

$$
\frac{\xi^{\prime}(s)}{\xi(s)}=\phi^{\prime}(s)+2 \frac{Z_{S, \chi}^{\prime}(s, \sigma)}{Z_{S, \chi}(s, \sigma)},
$$

so

$$
\begin{aligned}
N(t)=\frac{1}{2 \pi} \operatorname{Im}\left(\int_{C} \phi^{\prime}(s) d s\right)+ \\
\quad \frac{1}{\pi} \operatorname{Im}\left(\int_{C} \frac{Z_{S, \chi}^{\prime}(s, \sigma)}{Z_{S, \chi}(s, \sigma)} d s\right)+O(1) \\
=\frac{1}{2 \pi} \operatorname{Im}(\phi(\mathrm{i} t)-\phi(a)) \\
+\frac{1}{\pi} S(t)+O(1) \\
=\frac{1}{2 \pi} \operatorname{Im} \phi(\mathrm{i} t)+\frac{1}{\pi} S(t)-\frac{1}{2 \pi} \phi(a)+O(1) \\
=\frac{K}{2 \pi} \sum_{k=0}^{\frac{n}{2}-1}(-1)^{\frac{n}{2}-k} p_{n-2 k-1} \frac{t^{n-2 k}}{n-2 k} \\
\quad+\frac{1}{\pi} S(t)+O(1) .
\end{aligned}
$$

This completes the proof in this case. 
Suppose that $n$ is an odd integer.

Define,

$$
\xi(s)=(f(s))^{2} e^{\phi(s)},
$$

where

$$
\phi(s)=-2 \sum_{k=0}^{\frac{n-1}{2}} c_{\frac{2 k-n}{2}} \Gamma\left(\frac{2 k-n}{2}\right) s^{n-2 k},
$$

and $f(s)=Z_{S, \chi}(s, \sigma)$ (case $\left.(a)\right), f(s)=S_{\chi}(s, \sigma)$ (case $(b)$ ).

By Theorem 3.17 in $[4$, p. 116] (see also page 125), it follows that

$$
f(-s)=e^{\phi(s)} f(s) .
$$

Reasoning in exactly the same way as in the evendimensional case, we obtain that

$$
\xi(-s)=\xi(s)
$$

Assume that $t$ is chosen such that no singularity of $f(s)$ appears on the boundary of $R$ (now, $N_{0}=O(1)$ is the number of singularities of $f(s)$ on the real line).

By the identity given in the proof of Theorem 11 in [11, p. 319], we have that

$$
\begin{aligned}
& \phi\left(\sigma_{1}+\mathrm{i} t\right) \\
= & -2 \sum_{k=0}^{\frac{n-1}{2}} c_{\frac{2 k-n}{2}} \Gamma\left(\frac{2 k-n}{2}\right) \times \\
& \times \sum_{l=0}^{\frac{n-1}{2}-k}\left(\begin{array}{c}
n-2 k \\
2 l
\end{array}\right)(-1)^{l} \sigma_{1}^{n-2 k-2 l}|t|^{2 l} \\
= & -2 \mathrm{i} \sum_{k=0}^{\frac{n-1}{2}} c_{\frac{2 k-n}{2}} \Gamma\left(\frac{2 k-n}{2}\right) \times \\
\times & \sum_{l=1}^{\frac{n+1}{2}-k}\left(\begin{array}{c}
n-2 k \\
2 l-1
\end{array}\right)(-1)^{l-1} \sigma_{1}^{n-2 k-2 l+1} t^{2 l-1} .
\end{aligned}
$$

Now,

$$
\frac{\xi^{\prime}(s)}{\xi(s)}=\phi^{\prime}(s)+2 \frac{f^{\prime}(s)}{f(s)}
$$

so

$$
\begin{aligned}
& N(t) \\
= & \frac{1}{\pi} \sum_{k=0}^{\frac{n-1}{2}}(-1)^{\frac{n+1}{2}-k} c_{\frac{2 k-n}{2}} \Gamma\left(\frac{2 k-n}{2}\right) t^{n-2 k} \\
& +\frac{1}{\pi} S(t)+O(1) .
\end{aligned}
$$

This completes the proof.

Theorem 5. $S(t)=O\left(t^{n-1}(\log t)^{-1}\right)$.

Proof. Let $n$ be an even integer.

Firstly, we extend the definition of $S(t)$ to those values of $t$ for which it is a pole or zero of $Z_{S, \chi}(s, \sigma)$, by defining it to be

$$
\lim _{\varepsilon \rightarrow 0} \frac{1}{2}(S(t+\varepsilon)+S(t-\varepsilon)) .
$$

Then, we have

$$
S(t)=h(t)+O(1)
$$

where $h(t)$ is the variation of the argument of $Z_{S, \chi}(s, \sigma)$ along the segment from $a+\mathrm{i} t$ to it .

Proceeding in accordance with a custom, one easily concludes that

$$
\begin{aligned}
& h(t) \\
= & O\left(\int_{\partial S} \log \left|Z_{S, \chi}(w+\mathrm{i} t, \sigma)\right|+\right. \\
& \left.\log \left|Z_{S, \chi}(w-\mathrm{i} t, \sigma)\right| d w\right) \\
= & O\left(\sum_{i=1,2} \int_{\partial S} \log \left|Z_{i}(w+\mathrm{i} t)\right| d w+\right. \\
& \left.\int_{\partial S} \log \left|Z_{i}(w-\mathrm{i} t)\right| d w\right),
\end{aligned}
$$

where $S$ is the closed disc, centered at $a$, of radius $a$ $+\frac{1}{4}$. $O\left(t^{n-1}\right)$. 
From this point on, the improved bound $S(t)=$ $O\left(t^{n-1}(\log t)^{-1}\right)$ is obtained by the same reasoning as in the case $n=2$ [1, pp. 213-218].

If $n$ is an odd integer, then the proof of the assertion is quite the same, with the only difference that we have to distinguish between cases $f(s)=Z_{S, \chi}(s, \sigma)$ (case $(a)$ ), and $f(s)=S_{\chi}(s, \sigma)$ (case $(b)$ ).

This completes the proof.

Theorem 6. If $n$ is an even integer, then

$$
\begin{array}{r}
N(t)=\frac{\operatorname{dim}(\chi) \operatorname{vol}(Y)}{n T \operatorname{vol}\left(X_{d}\right)} t^{n}+ \\
O\left(t^{n-1}(\log t)^{-1}\right) .
\end{array}
$$

If $n$ is an odd integer, then

$$
\begin{aligned}
N(t)= & \frac{1}{\pi}(-1)^{\frac{n+1}{2}} \Gamma\left(-\frac{n}{2}\right) c_{-\frac{n}{2}} t^{n}+ \\
& O\left(t^{n-1}(\log t)^{-1}\right) .
\end{aligned}
$$

Proof. Let $n$ be an even integer.

By [4, p. 36]

$$
\frac{\chi(Y)}{\chi\left(X_{d}\right)}=(-1)^{\frac{n}{2}} \frac{\operatorname{vol}(Y)}{\operatorname{vol}\left(X_{d}\right)} .
$$

Now, the assertion is an immediate consequence of (10), Theorem 4, and Theorem 5.

More precisely, we deduce

$$
\begin{aligned}
N(t)= & \frac{\operatorname{dim}(\chi) \chi(Y)}{\chi\left(X_{d}\right) T} \times \\
& \times \sum_{k=0}^{\frac{n}{2}-1}(-1)^{\frac{n}{2}-k} p_{n-2 k-1} \frac{t^{n-2 k}}{n-2 k}+ \\
& \frac{1}{\pi} S(t)+O(1) \\
= & \frac{\operatorname{dim}(\chi) \chi(Y)}{\chi\left(X_{d}\right) T}(-1)^{\frac{n}{2}} p_{n-1} \frac{t^{n}}{n}+ \\
& \frac{\operatorname{dim}(\chi) \chi(Y)}{\chi\left(X_{d}\right) T} \times \\
& \times \sum_{k=1}^{\frac{n}{2}-1}(-1)^{\frac{n}{2}-k} p_{n-2 k-1} \frac{t^{n-2 k}}{n-2 k}+ \\
& \frac{1}{\pi} O\left(t^{n-1}(\log t)^{-1}\right)+O(1)
\end{aligned}
$$

$$
\begin{aligned}
= & \frac{\operatorname{dim}(\chi)}{T}(-1)^{\frac{n}{2}} \frac{\operatorname{vol}(Y)}{\operatorname{vol}\left(X_{d}\right)} \times \\
& \times(-1)^{\frac{n}{2}} \cdot 1 \cdot \frac{t^{n}}{n}+ \\
& O\left(t^{n-1}(\log t)^{-1}\right) \\
= & \frac{\operatorname{dim}(\chi) \operatorname{vol}(Y)}{n T \operatorname{vol}\left(X_{d}\right)} t^{n}+ \\
& O\left(t^{n-1}(\log t)^{-1}\right) .
\end{aligned}
$$

If $n$ is an odd integer, we apply Theorem 4 and Theorem 5, to obtain

$$
\begin{aligned}
& N(t) \\
= & \frac{1}{\pi} \sum_{k=0}^{\frac{n-1}{2}}(-1)^{\frac{n+1}{2}-k} c_{\frac{2 k-n}{2}} \Gamma\left(\frac{2 k-n}{2}\right) t^{n-2 k}+ \\
& \frac{1}{\pi} S(t)+O(1) \\
= & \frac{1}{\pi}(-1)^{\frac{n+1}{2}} c_{-\frac{n}{2}} \Gamma\left(-\frac{n}{2}\right) t^{n}+ \\
& \frac{1}{\pi} \sum_{k=1}^{\frac{n-1}{2}}(-1)^{\frac{n+1}{2}-k} \frac{c_{\frac{2 k-n}{2}} \Gamma\left(\frac{2 k-n}{2}\right) t^{n-2 k}+}{} \\
& \frac{1}{\pi} O\left(t^{n-1}(\log t)^{-1}\right)+O(1) \\
= & \frac{1}{\pi}(-1)^{\frac{n+1}{2}} \Gamma\left(-\frac{n}{2}\right) c_{-\frac{n}{2}} t^{n}+ \\
& O\left(t^{n-1}(\log t)^{-1}\right) .
\end{aligned}
$$

This completes the proof.

Theorem 7. Let $-\rho \leq a \leq b \leq \rho$. Then, there exists a constant $C$, such that

$$
N_{R}(t)=C t^{n}+O\left(t^{n-1}(\log t)^{-1}\right),
$$

where $N_{R}(t)$ denotes the number of singularities of $Z_{R, \chi}(s, \sigma)$ in the rectangle $a \leq \operatorname{Re}(s) \leq b, 0<$ $\operatorname{Im}(s)<t$.

Proof. Trivial consequence of Proposition 3.4 in [4, p. 99], and Theorem 6. 
Examples: Analogous formulas of the formulas derived in Theorem 6 and Theorem 7 are already known and are very well applied in literature for various settings of locally symmetric spaces. Let us recall some of them.

(a) In the classical arithmetic case, $N(T), T>$ 0 , denotes the number of zeros of the Riemann zeta function $\zeta(s)$ in the region $0 \leq \sigma \leq 1,0<t \leq T$. By Theorem 9.3 in [14, p. 212],

$$
\begin{aligned}
N(T)= & \frac{1}{2 \pi} T \log T-\frac{1+\log 2 \pi}{2 \pi} T+ \\
& \frac{7}{8}+S(T)+O\left(\frac{1}{T}\right),
\end{aligned}
$$

where $S(T)$ denotes the value of $\pi^{-1} \arg \zeta\left(\frac{1}{2}+\mathrm{i} T\right)$ obtained by continuous variation along the straight lines joining $2,2+\mathrm{i} T, \frac{1}{2}+\mathrm{i} T$, starting with the value 0 . As it is proven by Theorem 9.4 in [14, p. 214], $S(T)=O(\log T)$, so

$$
\begin{aligned}
N(T)= & \frac{1}{2 \pi} T \log T-\frac{1+\log 2 \pi}{2 \pi} T+ \\
& O(\log T) .
\end{aligned}
$$

(b) In the case of compact Riemannian surfaces (see section 1),

$$
N(T)=\frac{A}{2 \pi} T^{2}+4 S(T)+O(1),
$$

where $N(T)$ is the number of zeros of the Selberg zeta function $Z(s)$ in the rectangle $R$ given by $1-a$ $\leq \sigma \leq a,-T \leq t \leq T\left(1<a<\frac{5}{4}\right)$, and $S(T)$ is $\pi^{-1}$ times the valuation of the argument of $Z(s)$ along the vertical segment from $a$ to i $T$, plus the horizontal segment from $a+\mathrm{i} T$ to $\frac{1}{2}+\mathrm{i} T$. By Theorem 1 in $[1$, p. 216], $S(T)=O\left(T(\log T)^{-1}\right)$, so

$$
N(T)=\frac{A}{2 \pi} T^{2}+O\left(T(\log T)^{-1}\right) .
$$

(c) Hejhal [2, pp. 115-119], derived the same formula for $N(T)$ as in the case $(b)$. More precisely, he obtained that $N(T)=c T^{2}+S(T)+$ $E(T)$, where $c=2 \pi(g-1), E(T)=O(1), S(T)$
$=O\left(T(\log T)^{-1}\right)$. Here, $g \geq 2$ is the genus of the compact Riemann surface. Regarding this formula for $N(T)$, we point out the pages $1,41,115$, and 119 in [2]. Actually, it was pointed out by Randol [1, p. 210], that he and Hejhal derived similar results independently.

(d) Pavey [9, pp. 58-60], proved that $N(T)=$ $O\left(T^{D}\right)$, where $N(T), T>0$ is the number of poles and zeros of the corresponding Selberg zeta function (in the case of compact symmetric spaces formed as quotients of the Lie group $S L_{4}(\mathbb{R})$ ), and $D$ is the degree of the polynomial $G$ that appears in the functional equation of the Selberg zeta function. He proved that

$$
N(T)=\frac{1}{\pi} S(T)+O\left(T^{D}\right)+O(1),
$$

where $S(T)$ is the variation of the argument of the Selberg zeta function along the vertical segment from $a$ to $a+\mathrm{i} T$ plus the horizontal segment from $a+\mathrm{i} T$ to $\frac{1}{2}+\mathrm{i} T\left(1<a<\frac{5}{4}\right)$. It was proved that $S(T)$ $=O\left(T^{D}\right)$, so the formula $N(T)=O\left(T^{D}\right)$ was established. Regarding the applications of the formulas $N(T)$ 's, we point out that in the case $(a)$, the formula for $N(T)$ is applied in literature further on to derive the prime number theorem as well as some auxiliary results, in cases $(b)$ and $(c)$, it is used to obtain approximate formulas for the logarithmic derivative of the zeta functions of Selberg and Ruelle, and in the end, to improve the error terms in the prime geodesic theorem. In the case $(d), N(T)$ was also used as the main tool for obtaining of the corresponding prime geodesic theorem.

\section{Concluding remarks}

Precise estimates on the number of singularities of zeta functions represent an important tool which plays the key role in achieving more refined error terms in prime geodesic theorems.

Thus, some authors consider compact Riemann surfaces [15], [16], [17], higher-dimensional case [18], [19], quartic fields [12], [9], modular case [20], [21], [22].

However, an approach using the Selberg zeta function is not always sufficient to reach expected error terms in the prime geodesic theorem. Namely, the prime geodesic theorem states that

$$
\pi_{X}(x) \sim \frac{x^{d-1}}{(d-1) \log x},
$$


where $X$ is a $d$-dimensional locally symmetric space, and $\pi_{X}(x)$ is a function counting prime geodesics whose norm is not larger that $x$. The prime geodesic theorem (11) was proved by Gangolli [23] and DeGeorge [24] independently when $X$ is compact, and by Gangolli-Warner [8] when $X$ has a finite volume. Proofs of Gangolli and Gangolli-Warner are based on application of the corresponding Selberg zeta funcion. Similarly, Hejhal [2], [3], proved the prime geodesic theorem with the error terms for hyperbolic Rieman surfaces $X$

$$
\begin{aligned}
\pi_{X}(x)= & \sum_{\frac{3}{4}<s_{n} \leq 1} \operatorname{li}\left(x^{s_{n}}\right)+ \\
& O\left(x^{\frac{3}{4}}(\log x)^{-\frac{1}{2}}\right),
\end{aligned}
$$

where $s_{n}$ is a zero of the corresponding Selberg zeta function. So, Hejhal also applied the Selberg zeta function to derive the prime geodesic theorem, and, recalling that the leading term of $\operatorname{li}(x)$ is $\frac{x}{\log x}$, as $x$ $\rightarrow \infty$, it is easily seen that Hejhal's theorem (12) is a refinement of Gangolli's (Gangolli-Warner's) theorem (11) (although both of them are obtained via corresponding Selberg zeta function). Comparing (11) with (12), one could try to obtain an analogous error term in (11) as in (12) for a $d$-dimensional locally symmetric space $X$ of rank one. Of course, desired approach would be the one via Selberg zeta function. Namely, one may belive that the poles of the logarithmic derivative of the corresponding Selberg zeta function over the strip $\frac{1}{2}(d-1)<\operatorname{Re}(s) \leq d-1$ would provide the expected error terms. However, as explained in [19], this approach provides only the error terms corresponding to the poles of the logarithmic deriivative of the Selberg zeta function which are positioned in the strip $d-2 \leq \operatorname{Re}(s) \leq d-1$. Hence, the Selberg zeta function in this case is not sufficient to obtain the expected error terms for $d>3$. The same fact can also be seen in [23] and [8] in the view of the Tauberian theorem of Wiener-Ikehara. Thus, in case $d>3$, the meromorphic continuation of the Ruelle zeta function yields more satisfactory results [18], [19], [17].

As already noted, to achieve such results, one must obtain precise estimates on the number of singularities of zeta functions.

Though all terms after the leading one in the sum on the right-hand side of the expression in Theorem 4 are dominated by the order of $S(t)$, we expect that the precise knowledge of these might be useful in future investigations. Moreover, regarding the future work and the directions of the future research, we want to note that the estimates in this paper are derived under assumption that the attached space is compact. This fact enables us to use the Weyl asymptotic law (1) as a guarantee that our estimates could be derived at all. Unfortunately, in the non-compact case, analogous Weyl asymptotic laws are not available yet. More precisely, the only known such law is derived by Donnelly [25, p. 239] for finite volume symmetric spaces. In partiular, he proved that

$$
\limsup _{\lambda \rightarrow \infty} \frac{N(\lambda)}{\lambda^{\frac{d}{2}}} \leq(4 \pi)^{-\frac{d}{2}} \frac{\operatorname{vol}(X)}{\Gamma\left(\frac{d}{2}+1\right)}
$$

where $d=\operatorname{dim}(X), N(\lambda)$ is the number of linearly independent cuspidal eigenfunctions with eigenvalues less than $\lambda>0$, and $X$ is a non-compact locally symmetric space of finite volume. This estimate was applied in $[19$, p. 93] in the proof of the prime geodesic theorem in the case of real hyperbolic manifolds with cusps. As far as the author knows, analogous estimates are not available for the remaining non-compact locally symmetric spaces. Since such estimates are responsible for achieving more refined estimates of the logarithmic derivative of the corresponding zeta functions, and ultimately for obtaining improved error terms in the corresponding prime geodesic theorems, we shall concentrate our future research in this area into this direction.

The way we arrived at Theorem 6 is a Randoltype approach referred to in [5, p. 30].

In the case of compact Riemann surfaces, the estimates for $N(T)$ are applied in: [26, p. 751], [27, p. 3], [28, p. 74]. In the case of compact, locally symmetric spaces formed as quotients of the Lie group $S L_{4}(\mathbb{R})$, the approximate formulas for $N(T)$ are used in: [29, p. 23], [30, p. 8], [31, p. 6], [32, p. 40], [33, p. 58]. Finally, in general, in the case of compact locally symmetric spaces of real rank one, the applications of such formulas for $N(T)$ are found in: [34, p. 315], [35, p. 181], [36, p. 216].

\section{References:}

[1] B. Randol, The Riemann hypothesis for Selberg's zeta - function and the asymptotic behavior of eigenvalues of the Laplace operator, Trans. Amer. Math. Soc. 236, 1978, pp. 209-223.

[2] D. Hejhal, The Selberg trace formula for PSL $(2, \mathbb{R})$. Vol. I. Lecture Notes in Mathematics 548, Springer-Verlag, Berlin-Heidelberg 1976

[3] D. Hejhal, The Selberg Trace Formula for $\operatorname{PSL}(2, R)$, Springer-Verlag, Berlin 1983 
[4] U. Bunke and M. Olbrich, Selberg zeta and theta functions. A differential Operator Approach, Akademie-Verlag, Berlin 1995

[5] J. J. Duistermaat, J. A. C. Kolk, and V. S. Varadarajan, Spectra of compact locally symmetric manifolds of negative curvature, Invent. Math. 52, 1979, pp. 27-93.

[6] J. B. Conway, Functions of One Complex Variable, Springer-Verlag, New York-HeidelbergBerlin 1978

[7] R. Gangolli, Zeta functions of Selberg's type for compact space forms of symmetric spaces of rank one, Illinois J. Math. 21, 1977, pp. 1-42.

[8] R. Gangolli and G. Warner, Zeta functions of Selberg's type for some noncompact quotients of symmetric spaces of rank one, Nagoya Math. J. 78, 1980, pp. 1-44.

[9] M. Pavey, Class Numbers of Orders in Quartic Fields, University of Tubingen, Tubingen 2006

[10] M. Avdispahić and Dž. Gušić, Odrer of Selberg's and Ruelle's zeta functions for compact evendimensional locally symmetric spaces, J. Math. Anal. Appl. 413, 2014, pp. 525-531.

[11] M. Avdispahić and Dž. Gušić, On the length spectrum for compact locally symmetric spaces of real rank one, WSEAS Trans. on Math. 16, 2017, pp. 303-321.

[12] A. Deitmar and M. Pavey, A prime geodesic theorem for SL4, Ann. Glob. Anal. Geom. 33, 2008, pp. 161-205.

[13] M. Avdispahić and Dž. Gušić, Prime geodesic theorem for compact even- dimensional locally symmetric spaces of real rank one, Int. J. Pure Math. 4, 2017, pp. 26-36.

[14] E. C. Titchmarsh, The Theory of the Riemann Zeta-function, Claredon-Press, Oxford 1986

[15] H. Huber, Zur analytischen Theorie hyperbolischer Raumformen und Bewegungsgruppen. II, Math. Annalen 142, 1961, pp. 385-398.

[16] H. Huber, Zur analytischen Theorie hyperbolischer Raumformen und Bewegungsgruppen. II (Nachtrag zu Math. Annalen 142, 385-398, 1961), Math. Annalen 143, 1961, pp. 463-464.

[17] B. Randol, On the asymptotic distributon of closed geodesics on compact Riemann surfaces, Trans. Amer. Math. Soc. 233, 1977, pp. 241-247.

[18] M. Avdispahić and Dž. Gušić, On the error term in the prime geodesic theorem, Bull. Korean Math. Soc. 49, 2012, pp. 367-372.

[19] J. Park, Ruelle zeta function and prime geodesic theorem for hyperbolic manifolds with cusps, in: G. van Dijk, M. Wakayama (eds.), Casimir force, Casimir operators and Riemann hypothesis, de Gruyter, Berlin 2010, pp. 89-104.
[20] Y. Cai, Prime geodesic theorem for compact even- dimensional locally symmetric spaces of real rank one, J. Theor. Nom. Bord. 14, 2002, pp. 59-72.

[21] H. Iwaniec, Prime geodesic theorem, J. Reine Angew. Math. 349, 1984, pp. 136-158.

[22] K. Soundararajan and M. P. Young, The prime geodesic theorem, J. Reine Angew. Math. 676, 2013, pp. 105-120.

[23] R. Gangolli, The length spectrum of some compact manifolds of negative curvature, J. Diff. Geom. 12, 1977, pp. 403-426.

[24] D. L. DeGeorge, Length spectrum for compact locally symmetric spaces of strictly negative curvature, Ann. Sci. Ecole Norm. Sup. 10, 1977, pp. 133-152.

[25] H. Donnelly, On the cuspidal spectrum for finite volume symmetric spaces, J. Diff. Geom. 17, 1982, pp. 239-253.

[26] Dž. Gušić, Prime Geodesic Theorem for Compact Riemann Surfaces, Int. J. of Circuits, Systems and Signal Processing 13, 2019, pp. 747753.

[27] Dž. Gušić, On the remainder in the weighted length spectrum for strictly hyperbolic Fuchsian groups, in: MMCTSE 2020, Journal of Physics: Conference series, 1564 012015, pp. 1-8.

[28] Dž. Gušić, On Some Higher Order Counting Functions for $P S L(2, \mathbb{R})$, WSEAS Trans. on Systems and Control 15, 2020, pp. 73-80.

[29] Dž. Gušić, Approximate Formulas for Zeta Functions of Selberg's Type in Quotients of $S_{4}(\mathbb{R})$, Int. J. of Circuits, Systems and Signal Processing 14, 2020, pp. 21-27.

[30] Dž. Gušić, On the error term in the prime geodesic theorem for SL4, in: MMCTSE 2020, Journal of Physics: Conference series, 1564 012022, pp. 1-14.

[31] Dž. Gušić, On generalized length spectrum in quotients of $S L 4$, in: MMCTSE 2020, Journal of Physics: Conference series, 1564012023 , pp. 1-15.

[32] Dž. Gušić, On Some Classical and Weighted Estimates for SL4, WSEAS Trans. on Systems and Control 15, 2020, pp. 39-46.

[33] Dž. Gušić, On the Error Terms of Chebyshev Functions for SL4, WSEAS Trans. on Systems and Control 15, 2020, pp. 57-63.

[34] M. Avdispahić and Dž. Gušić, On the logarithmic derivative of zeta functions for compact even-dimensional locally symmetric spaces of real rank one, Mathematica Slovaca 69, 2019, pp. 311-320. 
[35] Dž. Gušić, On the Logarithmic Derivative of Zeta Functions for Compact, Odd-dimensional Hyperbolic Spaces, WSEAS Trans. on Math. 18, 2019, pp. 176-184.
[36] Dž. Gušić, On the Length Spectrum for Compact, Odd-dimensional, Real Hyperbolic Spaces, WSEAS Trans. on Math. 18, 2019, pp. 211-222.

Creative Commons Attribution License 4.0 (Attribution 4.0 International, CC BY 4.0)

This article is published under the terms of the Creative Commons Attribution License 4.0

https://creativecommons.org/licenses/by/4.0/deed.en_US 